\title{
Right Posteromedial Middle Peripheral Zone of Prostate
}

National Cancer Institute

\section{Source}

National Cancer Institute. Right Posteromedial Middle Peripheral Zone of Prostate. NCI

Thesaurus. Code $\mathrm{C} 128608$.

The region of the prostate that is located on the anatomical right side of the posteromedial portion of the middle division of the peripheral zone. 